\title{
Molybdenum and rhenium carbonyl complexes containing thiolato ligands
}

Purificación Cañadas, ${ }^{a}$ Sina Ziegler, ${ }^{a}$ Sergio Fombona, ${ }^{a}$ Eva Hevia,${ }^{b}$ Daniel Miguel, ${ }^{c}$ Julio Pérez,,${ }^{a, d}$ and Lucía Riera ${ }^{d^{*}}$

$a$ Departamento de Química Orgánica e Inorgánica. Universidad de Oviedo. Julián Clavería, 8, 33006 Oviedo (Spain).

$b$ WestCHEM, Department of Pure and Applied Chemistry. University of Strathclyde. 295 Cathedral Street, G1 1XL Glasgow (UK)

c GIR MIOMeT-IU CINQUIMA/Química Inorgánica. Facultad de Ciencias. Universidad de Valladolid. C/ Paseo de Belén 7. 47011 Valladolid (Spain).

$d$ Centro de Investigación en Nanomateriales y Nanotecnología (CINN). CSIC-Universidad de OviedoPrincipado de Asturias. Avenida de la Vega 4-6, 33940 El Entrego (Spain). Corresponding author email address: 1.riera@cinn.es (L. Riera) 


\section{ABSTRACT}

The reaction of $f a c-\left[\mathrm{M}(\mathrm{OTf})(\mathrm{CO})_{3}(\mathrm{~N}-\mathrm{N})\right](\mathrm{M}=\mathrm{Mn}, \mathrm{Re} ; \mathrm{N}-\mathrm{N}=2$ 2,2'-bipyridine, 1,10-phenanthroline) complexes with in situ generated LiSEt afforded immediately, in THF at low temperature, the corresponding mononuclear terminal thiolato complexes $f a c-\left[\mathrm{M}(\mathrm{SEt})(\mathrm{CO})_{3}(\mathrm{~N}-\mathrm{N})\right](\mathbf{1 - 2 a}, \mathbf{b})$. Using an analogous synthetic strategy, tungsten and molybdenum (II) alkyl- and arylthiolatos of formula cis$\left[\mathrm{M}(\mathrm{SR})\left(\eta^{3}\right.\right.$-allyl $\left.)(\mathrm{CO})_{2}(\mathrm{~N}-\mathrm{N})\right](\mathbf{3 - 6 a}, \mathbf{b})$ have been obtained. Preliminary reactions of the more reactive molybdenum thiolato complexes towards dimethylacetylenedicarboxylate led to $Z$-alkenyl products $(\mathbf{7 a}, \mathbf{b})$, resulting from the insertion of the organic unsaturated electrophile into the Mo-S bond. For each new type of compound, one representative has been characterized in the solid state by X-ray diffraction.

\section{Keywords}

carbonyl complexes $\cdot$ insertion reactions $\cdot$ mononuclear thiolatos $\cdot$ molybdenum $\cdot$ rhenium

\section{Introduction}

Thiolato (SR) ligands have been extensively used since the very beginnings of coordination chemistry with both main group and transition metals, showing different bonding modes, and displaying a marked tendency to form bimetallic complexes and clusters in which the thiolato ligands bridge two or more metal centers.[1-3] Thiolato complexes are of particular relevance in bioinorganic chemistry,[4-6] as many enzymes feature metal coordination by the thiolato groups of cysteine residues; in medicine, gold thiolatos are of importance as drugs, particularly in arthritis therapy, $[7,8]$ and volatile molecular thiolatos have been used as starting materials for chemical vapor deposition (CVD) of metal or sulfide layers.[9, 10]

The behavior of organosulfur metal compounds has been extensively studied and is expected to be similar to those on the surface of catalysts employed in hydrodesulfurization (HDS), a process used to remove sulfur-containing impurities from crude petroleum in order to minimize environment pollution, and the poisoning of the catalysts used for subsequent transformations. [11-14] Although the mechanism of the catalytic process is still unknown, coordination of the SR group to the metal is believed to be an essential step. [15] The most commonly used hydrotreating catalysts are composed of 
a mixture of a group 6 element (Mo or W) and a "promoter" element (Co or Ni) deposited on a support, typically $\mathrm{Al}_{2} \mathrm{O}_{3}$.

Organometallic compounds can provide well-defined structural models displaying properties that can be used to elucidate those of complex catalysts, and, similarly, the types of reactions exhibited by organometallic compounds are often informative in gaining insights into the HDS process.[16-22] In metal carbonyl complexes the thiolato group is generally found acting as a bridging ligand, linked to two or three metal atoms. Complexes with terminal thiolato ligands are also known, but they are often unstable towards $\mathrm{CO}$ loss and subsequent formation of polymetallic species by formation of thiolato bridges.

We reported years ago a series of $\left[\mathrm{MX}\left(\eta^{3}\right.\right.$-allyl $\left.)(\mathrm{CO})_{2}(\mathrm{~N}-\mathrm{N})\right](\mathrm{M}=\mathrm{Mo}, \mathrm{W})$ and $\left[\mathrm{MX}(\mathrm{CO})_{3}(\mathrm{~N}-\mathrm{N})\right](\mathrm{M}=$ Mn, Re) with $X=$ alkyl,[23] alkynyl,[24] alkoxo[25, 26] and amido[27, 28] complexes, in which $\mathrm{N}-\mathrm{N}=$ 2,2'-bipyridine (bipy) or 1,10 phenanthroline (phen). These organometallic fragments have shown their stability (reactions of substitution of CO ligands were not found), the ease of their preparation, the convenience of displaying CO ligands (to serve as a spectroscopic IR handle) and a high redox stability. In addition, these new species showed a rich reactivity towards some unsaturated organic electrophiles. The work reported herein includes the synthesis and characterization of thiolato compounds of these allyldicarbonyl and fac-tricarbonyl fragments, and a preliminary study of the reactivity of the molybdenum species towards selected unsaturated organic electrophiles.

\section{Experimental Section}

\subsection{General procedures}

All manipulations were carried out under a nitrogen atmosphere using Schlenk techniques. Solvents were distilled from $\mathrm{Na}$ (hexane), $\mathrm{Na} /$ benzophenone (THF) and $\mathrm{CaH}_{2}\left(\mathrm{CH}_{2} \mathrm{Cl}_{2}\right)$. $\mathrm{Compounds}\left[\mathrm{MoCl}\left(\eta^{3}-\right.\right.$ allyl $\left.)(\mathrm{CO})_{2}(\mathrm{~N}-\mathrm{N})\right](\mathrm{N}-\mathrm{N}=$ bipy, phen $),[29]\left[\operatorname{Re}(\mathrm{OTf})(\mathrm{CO})_{3}(\mathrm{~N}-\mathrm{N})\right](\mathrm{N}-\mathrm{N}=$ bipy, phen) [25, 30] and $\left[\mathrm{Mn}(\mathrm{OTf})(\mathrm{CO})_{3}\right.$ (bipy)] [31] were prepared as previously reported. Deuterated dichloromethane (Cambridge Isotope Laboratories, Inc.) was stored under nitrogen in a Young tube and used without further purification. ${ }^{1} \mathrm{H}$ NMR and ${ }^{13} \mathrm{C}$ NMR spectra were recorded on a Bruker Avance 300, DPX-300 or Avance 400 spectrometer. NMR spectra are referred to the internal residual solvent peak for ${ }^{1} \mathrm{H}$ and ${ }^{13} \mathrm{C}\left\{{ }^{1} \mathrm{H}\right\}$ NMR. IR solution spectra were obtained in a Perkin-Elmer FT 1720-X spectrometer using 0.2 mm. $\mathrm{CaF}_{2}$ cells. NMR samples were prepared under nitrogen using Kontes manifolds purchased from Aldrich. 


\subsection{X-ray structure determinations}

General Description: For Compounds 2a and 4b. A crystal was attached to a glass fiber and transferred to a Bruker AXS SMART 1000 diffractometer with graphite monochromatized Mo Ka Xradiation and a CCD area detector. A hemisphere of the reciprocal space was collected up to $2 \theta=48.6^{\circ}$. Raw frame data were integrated with the SAINT[32] program. An empirical absorption correction was applied with the program SADABS.[33] For compound 7b: Crystal data were collected on an Oxford Diffraction Xcalibur Nova single crystal diffractometer, using $\mathrm{Cu}-\mathrm{K} \alpha$ radiation ( $\lambda=$ $1.5418 \AA$ ) at $100(2) \mathrm{K}$. Images were collected at a $65 \mathrm{~mm}$ fixed crystal-detector distance, using the oscillation method, with $1^{\circ}$ oscillation and variable exposure time per image (4-16 s). Data reduction and cell refinement was performed with the program CrysAlis ${ }^{\text {Pro }}$ RED.[34] An empirical absorption correction was applied using the SCALE3 ABSPACK. [34] All the structures were solved using direct methods and refined by Full-Matrix-Least-Squares against $\mathrm{F}^{2}$ with SHELX[35] under OLEX2.[36] The non-hydrogen atoms were refined anisotropically and hydrogen atoms were placed at idealised positions and refined using the riding model. Molecular graphics were made with ORTEP 3.[37] In the structure of 2a an incipient disorder was found to affect the thiolato ethyl group and one carbonyl ligand, but it could not be modelled satisfactorily. The thiolato group was refined with a constrained geometry, and the carbon atom $\mathrm{C}(3)$ could not be refined anisotropically. In the structure of $\mathbf{4 b}$, a short contact between methyl $\mathrm{H}$ atoms is probably an artifact which arises from a misplacement failure of the riding model. Crystal and refinement data are presented in Table 1. 
Table 1. Crystal data and refinement details for complexes $\mathbf{2 a}, \mathbf{4 b}$ and $\mathbf{7 b}$.

\begin{tabular}{|c|c|c|c|}
\hline compound & $2 a$ & $4 \mathbf{b}$ & $7 \mathrm{~b}$ \\
\hline formula & $\mathrm{C}_{15} \mathrm{H}_{13} \mathrm{ReSO}_{3} \mathrm{~N}_{2}$ & $\mathrm{MoSN}_{2} \mathrm{C}_{20} \mathrm{O}_{2} \mathrm{H}_{20}$ & $\mathrm{C}_{27} \mathrm{H}_{28} \mathrm{~N}_{2} \mathrm{O}_{6} \mathrm{SMoCl}_{2}$ \\
\hline$F_{\mathrm{w}}$ & 487.53 & 448.38 & 675.41 \\
\hline$T[\mathrm{~K}]$ & 293(2) & $293(2)$ & $150.00(10)$ \\
\hline crystal system & monoclinic & monoclinic & monoclinic \\
\hline space group & $\mathrm{P} 2_{1} / \mathrm{c}$ & $\mathrm{C} 2 / \mathrm{c}$ & $\mathrm{P} 2_{1} / \mathrm{n}$ \\
\hline$a[\AA]$ & $16.214(2)$ & $25.954(8)$ & $11.7120(7)$ \\
\hline$b[\AA]$ & $13.3338(17)$ & $11.406(3)$ & $14.1188(7)$ \\
\hline$c[\AA]$ & $16.042(2)$ & $13.583(4)$ & $18.0146(11)$ \\
\hline$\alpha\left[^{\circ}\right]$ & 90 & 90 & 90 \\
\hline$\beta\left[^{\circ}\right]$ & $109.801(2)$ & $105.891(5)$ & $96.253(5)$ \\
\hline$\gamma\left[{ }^{0}\right]$ & 90 & 90 & 90 \\
\hline$V\left[\AA^{3}\right]$ & $3263.1(7)$ & $3867.3(19)$ & 2961.2(3) \\
\hline$Z$ & 8 & 8 & 4 \\
\hline$\rho_{\text {calc }}\left[\mathrm{gcm}^{-3}\right]$ & 1.985 & 1.5400 & 1.515 \\
\hline$\mu\left[\mathrm{mm}^{-1}\right]$ & 7.588 & 0.802 & 6.304 \\
\hline$F(000)$ & 1856.0 & 1824.0 & 1376.0 \\
\hline cristal size $[\mathrm{mm}]$ & $0.18 \times 0.14 \times 0.12$ & $0.29 \times 0.21 \times 0.13$ & $0.24 \times 0.14 \times 0.05$ \\
\hline radiation & $\operatorname{MoK} \alpha(\lambda=0.71073)$ & $\operatorname{MoK} \alpha(\lambda=0.71073)$ & $\mathrm{CuK} \alpha(\lambda=1.54184)$ \\
\hline $2 \theta$ scan range $\left[{ }^{\circ}\right]$ & 2.67 to 46.534 & 3.264 to 47 & 7.974 to 141.192 \\
\hline no refletions collected & 14368 & 8419 & 14002 \\
\hline no independent reflections & $\begin{array}{l}4668\left[R_{\text {int }}=0.0569,\right. \\
\left.R_{\text {sigma }}=0.0524\right]\end{array}$ & $\begin{array}{l}2816\left[R_{\text {int }}=0.0210,\right. \\
\left.R_{\text {sigma }}=0.0209\right]\end{array}$ & $\begin{array}{l}5446\left[R_{\text {int }}=0.1002,\right. \\
\left.R_{\text {sigma }}=0.0948\right]\end{array}$ \\
\hline data/restrains/parameters & $4668 / 28 / 394$ & $2816 / 22 / 236$ & $5446 / 0 / 352$ \\
\hline goodness-of-fit on $F^{2}$ & 1.003 & 1.078 & 1.639 \\
\hline$R_{1} / R_{w 2}[I>2 \sigma(I)]$ & $\mathrm{R}_{1}=0.0818, \mathrm{wR}_{2}=0.1990$ & $\mathrm{R}_{1}=0.0343, \mathrm{wR}_{2}=0.0974$ & $\begin{array}{l}\mathrm{R}_{1}=0.0870, w \mathrm{R}_{2}= \\
0.2403\end{array}$ \\
\hline$R_{1} / R_{w 2}[$ all data $]$ & $\mathrm{R}_{1}=0.1051, \mathrm{wR}_{2}=0.2229$ & $\mathrm{R}_{1}=0.0436, \mathrm{wR}_{2}=0.1031$ & $\begin{array}{l}\mathrm{R}_{1}=0.1342, \quad w \mathrm{R}_{2}= \\
0.2823\end{array}$ \\
\hline Largest diff. peak/hole [e $\AA^{-3}$ ] & $10.50 /-2.85$ & $0.52 /-0.61$ & $1.31 /-1.68$ \\
\hline
\end{tabular}

\subsection{Synthesis and characterization}

$\left[\mathbf{M n}\left(\mathbf{S C H}_{2} \mathbf{C H}_{3}\right)(\mathbf{C O})_{3}(\mathbf{b i p y})\right](\mathbf{1}) . n \mathrm{BuLi}(0.230 \mathrm{~mL}$ of a $1.6 \mathrm{M}$ solution in hexane, $0.372 \mathrm{mmol})$ was added to a solution of EtSH $(28 \mu \mathrm{L}, 0.372 \mathrm{mmol})$ in THF $(10 \mathrm{~mL})$ at $-78{ }^{\circ} \mathrm{C}$. The resulting colorless solution was transferred using a cannula and nitrogen pressure to a solution of $\left[\mathrm{Mn}(\mathrm{OTf})(\mathrm{CO})_{3}(\right.$ bipy $\left.)\right]$ $(0.150 \mathrm{~g}, 0.338 \mathrm{mmol})$ in THF $(15 \mathrm{~mL})$ previously cooled to $-78{ }^{\circ} \mathrm{C}$. The color of the solution changed immediately from yellow to red. The solvent was evaporated to dryness under reduced pressure and the solid was extracted with $\mathrm{CH}_{2} \mathrm{Cl}_{2}(25 \mathrm{~mL})$. The resulting solution was filtered off the white solid (LiOTf) via cannula, concentrated under reduced pressure to a volume of $10 \mathrm{~mL}$, and addition of hexane caused the precipitation of compound $\mathbf{1}$ as a red solid, which was washed with hexane $(2 \times 15$ $\mathrm{mL})$ and dried. Yield: $0.086 \mathrm{~g}(72 \%)$. IR (THF, $\left.\mathrm{cm}^{-1}\right):$ 2003(vs), 1912(s), 1902(s) ( $\left.\mathrm{v}_{\mathrm{CO}}\right) .{ }^{1} \mathrm{H}$ NMR $\left(\mathrm{CD}_{2} \mathrm{Cl}_{2}\right): 9.10,8.11,7.95$ and 7.43 [m, 2H each, bipy], 2.37 [m, 2H, Mn-SCH $\left.\mathrm{CH}_{3}\right], 1.11[\mathrm{~m}, 3 \mathrm{H}, \mathrm{Mn}-$ $\left.\mathrm{SCH}_{2} \mathrm{CH}_{3}\right] .{ }^{13} \mathrm{C}\left\{{ }^{1} \mathrm{H}\right\} \mathrm{NMR}\left(\mathrm{CD}_{2} \mathrm{Cl}_{2}\right): 226.5$ [2CO], 212.2 [CO], 156.5, 154.7, 138.1, 127.2 and 124.9 
[bipy], 26.4 [Mn- $\mathrm{SCH}_{2} \mathrm{CH}_{3}$ ], $21.08 \mathrm{Mn}-\mathrm{SCH}_{2} \mathrm{CH}_{3}$ ]. Anal. Calcd. For $\mathrm{C}_{15} \mathrm{H}_{13} \mathrm{MnN}_{2} \mathrm{O}_{3} \mathrm{~S}$ : C, 50.56; H, 3.67; N, 7.86. Found: C, 50.32; H, 3.59; N, 7.81.

$\left[\operatorname{Re}\left(\mathrm{SCH}_{2} \mathrm{CH}_{3}\right)(\mathbf{C O})_{3}(\right.$ bipy $\left.)\right](\mathbf{2 a})$. Compound 2a was prepared as described above for 1, starting from $\left[\operatorname{Re}(\mathrm{OTf})(\mathrm{CO})_{3}(\right.$ bipy $\left.)\right](0.050 \mathrm{~g}, 0.087 \mathrm{mmol}), n \mathrm{BuLi}(65 \mu \mathrm{L}$ of a $1.6 \mathrm{M}$ solution in hexane, 0.104 $\mathrm{mmol})$ and EtSH $(8 \mu \mathrm{L}, 0.104 \mathrm{mmol})$ in THF $(10 \mathrm{~mL})$ at $-78^{\circ} \mathrm{C}$. Slow diffusion of hexane $(25 \mathrm{~mL})$ into a concentrated solution of $\mathbf{2 a}$ in $\mathrm{CH}_{2} \mathrm{Cl}_{2}(10 \mathrm{~mL})$ afforded red crystals of $\mathbf{2 a}$, one of which was used for the X-ray analysis. Yield: $0.032 \mathrm{~g}(72 \%)$. IR (THF, $\left.\mathrm{cm}^{-1}\right):$ 2003(vs), 1902(s), 1886(s) (v $\left.{ }_{\mathrm{CO}}\right) .{ }^{1} \mathrm{H}$ NMR $\left(\mathrm{CD}_{2} \mathrm{Cl}_{2}\right): 9.00,8.22,8.03$ and 7.50 [m, 2H each, bipy], 2.53 [q $\left.(J=7.4 \mathrm{~Hz}), 2 \mathrm{H}, \mathrm{Re}-\mathrm{SCH}_{2} \mathrm{CH}_{3}\right], 1.04[\mathrm{t}$ $\left.(J=7.4 \mathrm{~Hz}), 3 \mathrm{H}, \mathrm{Re}-\mathrm{SCH}_{2} \mathrm{CH}_{3}\right] .{ }^{13} \mathrm{C}\left\{{ }^{1} \mathrm{H}\right\} \mathrm{NMR}\left(\mathrm{CD}_{2} \mathrm{Cl}_{2}\right): 200.6$ [2CO], $191.3[C \mathrm{O}], 155.1,153.2$, 138.8, 127.1 and 123.5 [bipy], 25.4 [Re- $\left.\mathrm{SCH}_{2} \mathrm{CH}_{3}\right], 21.4$ [Re- $\left.\mathrm{SCH}_{2} \mathrm{CH}_{3}\right]$. Anal. Calcd. For $\mathrm{C}_{15} \mathrm{H}_{13} \mathrm{~N}_{2} \mathrm{O}_{3} \operatorname{ReS}:$ C, 36.95; H, 2.69; N, 5.75. Found: C, 36.68; H, 2.99; N, 5.60.

$\left[\operatorname{Re}\left(\mathrm{SCH}_{2} \mathrm{CH}_{3}\right)(\mathbf{C O})_{3}(\right.$ phen $\left.)\right](2 \mathrm{~b})$. Compound $\mathbf{2 b}$ was prepared as described above for $\mathbf{1}$, starting from $\left[\operatorname{Re}(\mathrm{OTf})(\mathrm{CO})_{3}(\right.$ phen $\left.)\right](0.068 \mathrm{~g}, 0.113 \mathrm{mmol}), n \mathrm{BuLi}(85 \mu \mathrm{L}$ of a $1.6 \mathrm{M}$ solution in hexane, 0.136 $\mathrm{mmol})$ and $\mathrm{EtSH}(9 \mu \mathrm{L}, 0.136 \mathrm{mmol})$ in $\mathrm{THF}(10 \mathrm{~mL})$ at $-78^{\circ} \mathrm{C}$. Compound $\mathbf{2 b}$ was obtained as a dark green microcrystalline solid. Yield: $0.030 \mathrm{~g}(52 \%)$. IR (THF, $\left.\mathrm{cm}^{-1}\right)$ : 2005(vs), 1905(s), 1888(s) ( $\left.\mathrm{v}_{\mathrm{CO}}\right)$. ${ }^{1} \mathrm{H}$ NMR $\left(\mathrm{CD}_{2} \mathrm{Cl}_{2}\right): 9.34,8.56,8.03$ and $7.86[\mathrm{~m}, 2 \mathrm{H}$ each, phen], $2.50[\mathrm{q}(J=7.4 \mathrm{~Hz}), 2 \mathrm{H}, \mathrm{Re}-$ $\left.\mathrm{SCH}_{2} \mathrm{CH}_{3}\right], 0.99$ [t $\left.(J=7.4 \mathrm{~Hz}), 3 \mathrm{H}, \mathrm{Re}-\mathrm{SCH}_{2} \mathrm{CH}_{3}\right] .{ }^{13} \mathrm{C}\left\{{ }^{1} \mathrm{H}\right\} \mathrm{NMR}\left(\mathrm{CD}_{2} \mathrm{Cl}_{2}\right): 200.4$ [2CO], 191.3 [CO], 153.1, 146.6, 137.9, 131.0, 127.8 and 125.9 [phen], 25.3 [Re- $\mathrm{SCH}_{2} \mathrm{CH}_{3}$ ], 21.4 [Re-SCH $\mathrm{CH}_{3}$ ]. Anal. Calcd. For $\mathrm{C}_{17} \mathrm{H}_{13} \mathrm{~N}_{2} \mathrm{O}_{3}$ ReS: C, 39.91; H, 2.56; N, 5.48. Found: C, 39.69; H, 2.82; N, 5.27.

$\left[\mathrm{Mo}\left(\mathbf{S C H}_{2} \mathbf{C H}_{3}\right)\left(\eta^{3}-\mathbf{C}_{3} \mathbf{H}_{5}\right)(\mathbf{C O})_{2}(\right.$ phen $\left.)\right](3) . n \mathrm{BuLi}(0.16 \mathrm{~mL}$ of a $1.6 \mathrm{M}$ solution in hexane, 0.250 mmol) was added to a solution of EtSH $(18 \mu \mathrm{L}, 0.250 \mathrm{mmol})$ in THF $(10 \mathrm{~mL})$ at $-78{ }^{\circ} \mathrm{C}$. The resulting colorless solution was transferred using a cannula and nitrogen pressure to a solution of $\left[\mathrm{Mo}\left(\eta^{3}-\right.\right.$ $\left.\mathrm{C}_{3} \mathrm{H}_{5}\right)(\mathrm{OTf})(\mathrm{CO})_{2}($ phen $\left.)\right](0.080 \mathrm{~g}, 0.208 \mathrm{mmol})$ in THF $(15 \mathrm{~mL})$ previously cooled to $-78^{\circ} \mathrm{C}$. The red color of the solution turned darker immediately. The solvent was evaporated to dryness under reduced pressure and the solid was extracted with $\mathrm{CH}_{2} \mathrm{Cl}_{2}(25 \mathrm{~mL})$. The resulting solution was filtered off the white solid (LiOTf) via cannula, concentrated under reduced pressure to a volume of $10 \mathrm{~mL}$, and addition of hexane caused the precipitation of compound $\mathbf{3}$ as a dark red solid, that was washed with hexane $(2 \times 15 \mathrm{~mL})$ and dried. Yield: $0.066 \mathrm{~g}(78 \%)$. IR $\left(\mathrm{CH}_{2} \mathrm{Cl}_{2}, \mathrm{~cm}^{-1}\right): 1934(\mathrm{vs}), 1850(\mathrm{~s})\left(v_{\mathrm{CO}}\right) .{ }^{1} \mathrm{H}$ $\operatorname{NMR}\left(\mathrm{CD}_{2} \mathrm{Cl}_{2}\right): 9.05,8.43,7.94$ and 7.77 [m, 2H each, phen], 3.27 [q $\left.(J=7.4 \mathrm{~Hz}), 2 \mathrm{H}, \mathrm{Mo}-\mathrm{SCH}_{2} \mathrm{CH}_{3}\right]$, $3.11\left[\mathrm{~s}_{\mathrm{br}}, 2 \mathrm{H}, \mathrm{H}_{s y n}\right], 1.61\left[\mathrm{~m}, 1 \mathrm{H}, \mathrm{H}_{\mathrm{c}}\right], 1.52\left[\mathrm{~d}(\mathrm{~J}=8.7 \mathrm{~Hz}), 2 \mathrm{H}, \mathrm{H}_{\text {anti }}\right], 1.20[\mathrm{t}(\mathrm{J}=7.4 \mathrm{~Hz}), 3 \mathrm{H}, \mathrm{Mo}-$ 
$\left.\mathrm{SCH}_{2} \mathrm{CH}_{3}\right] .{ }^{13} \mathrm{C}\left\{{ }^{1} \mathrm{H}\right\}$ NMR $\left(\mathrm{CD}_{2} \mathrm{Cl}_{2}\right): 239.5$ [CO], 152.2, 137.1, 130.2, 127.6, 127.4 and 125.0 [phen], $77.7\left[\mathrm{C}^{2}\right.$ of $\left.\eta^{3}-\mathrm{C}_{3} \mathrm{H}_{5}\right], 53.5\left[\mathrm{C}^{1}\right.$ and $\mathrm{C}^{3}$ of $\eta^{3}-\mathrm{C}_{3} \mathrm{H}_{5}$ ], 28.4 [Mo- $\mathrm{SCH}_{2} \mathrm{CH}_{3}$ ], 20.9 [Mo- $\left.\mathrm{SCH}_{2} \mathrm{CH}_{3}\right]$. Anal. Calcd. For $\mathrm{C}_{19} \mathrm{H}_{18} \mathrm{MoN}_{2} \mathrm{O}_{2} \mathrm{~S}$ : C, 52.53; H, 4.18; N, 6.45. Found: C, 52.13; H, 4.27; N, 6.47.

$\left[\mathrm{Mo}\left(\mathrm{SCH}_{2} \mathrm{CH}_{3}\right)\left(\eta^{3}-\mathrm{C}_{4} \mathrm{H}_{7}\right)(\mathrm{CO})_{2}(\mathrm{bipy})\right](4 a)$. Compound 4a was prepared as described above for the synthesis of $\mathbf{3}$, starting from $\left[\mathrm{MoCl}\left(\eta^{3}-\mathrm{C}_{4} \mathrm{H}_{7}\right)(\mathrm{CO})_{2}\right.$ (bipy)] $(0.085 \mathrm{~g}, 0.213 \mathrm{mmol}), n \mathrm{BuLi}(0.16 \mathrm{~mL}$ of a 1.6 M solution in hexane, $0.256 \mathrm{mmol})$ and $\mathrm{EtSH}(19 \mu \mathrm{L}, 0.256 \mathrm{mmol})$. Compound $4 \mathbf{a}$ was obtained as a dark red solid, which was washed with hexane $(2 \times 15 \mathrm{~mL})$ and dried. Yield: $0.076 \mathrm{~g}(84 \%)$. IR $\left(\mathrm{CH}_{2} \mathrm{Cl}_{2}, \mathrm{~cm}^{-1}\right): 1933(\mathrm{vs}), 1855(\mathrm{~s})\left(v_{\mathrm{CO}}\right) .{ }^{1} \mathrm{H} \mathrm{NMR}\left(\mathrm{CD}_{2} \mathrm{Cl}_{2}\right): 8.64,8.10,7.93$ and 7.43 [m, $2 \mathrm{H}$ each, bipy], $3.22\left[\mathrm{q}(\mathrm{J}=7.2 \mathrm{~Hz}), 2 \mathrm{H}, \mathrm{Mo}-\mathrm{SCH}_{2} \mathrm{CH}_{3}\right], 2.73\left[\mathrm{~s}, 2 \mathrm{H}, \mathrm{H}_{\text {syn }}\right], 1.52\left[\mathrm{~s}, 2 \mathrm{H}, \mathrm{H}_{\text {anti }}\right], 1.23[\mathrm{t}(\mathrm{J}=7.2 \mathrm{~Hz})$, $\left.3 \mathrm{H}, \mathrm{Mo}-\mathrm{SCH}_{2} \mathrm{CH}_{3}\right], 0.93$ [s, 3H, $\mathrm{CH}_{3}$ of $\left.\eta^{3}-\mathrm{C}_{4} \mathrm{H}_{7}\right] .{ }^{13} \mathrm{C}\left\{{ }^{1} \mathrm{H}\right\} \mathrm{NMR}\left(\mathrm{CD}_{2} \mathrm{Cl}_{2}\right): 231.9[C \mathrm{O}], 153.2,152.4$, 137.8, 126.1 and 122.5 [bipy], 88.2 [C $\mathrm{C}^{2}$ of $\eta^{3}-\mathrm{C}_{4} \mathrm{H}_{7}$ ], 28.5 [Mo- $\mathrm{SCH}_{2} \mathrm{CH}_{3}$ ], 21.1 [Mo- $\left.\mathrm{SCH}_{2} \mathrm{CH}_{3}\right], 19.4$ $\left[\mathrm{CH}_{3}\right.$ of $\left.\eta^{3}-\mathrm{C}_{4} \mathrm{H}_{7}\right]$. The signals corresponding to $\mathrm{C}^{1}$ and $\mathrm{C}^{3}$ of the methallyl ligand are overlapped with the solvent residual peak, a fact clearly indicated by a 2D-HSQC experiment. Anal. Calcd. For $\mathrm{C}_{18} \mathrm{H}_{20} \mathrm{MoN}_{2} \mathrm{O}_{2} \mathrm{~S}: \mathrm{C}, 50.94 ; \mathrm{H}, 4.75 ; \mathrm{N}, 6.60$. Found: C, 51.07; H, 5.08; N, 6.99.

$\left[\mathrm{Mo}\left(\mathrm{SCH}_{2} \mathrm{CH}_{3}\right)\left(\eta^{3}-\mathrm{C}_{4} \mathrm{H}_{7}\right)(\mathrm{CO})_{2}(\right.$ phen $\left.)\right](4 \mathrm{~b})$. Compound $4 \mathrm{~b}$ was prepared as described above for $\mathbf{3}$, starting from $\left[\mathrm{MoCl}\left(\eta^{3}-\mathrm{C}_{4} \mathrm{H}_{7}\right)(\mathrm{CO})_{2}(\right.$ phen $\left.)\right](0.045 \mathrm{~g}, 0.106 \mathrm{mmol}), n \mathrm{BuLi}(80 \mu \mathrm{L}$ of a $1.6 \mathrm{M}$ solution in hexane, $0.128 \mathrm{mmol})$ and $\mathrm{EtSH}(9 \mu \mathrm{L}, 0.128 \mathrm{mmol})$. Compound $\mathbf{4 b}$ was obtained as dark red crystals obtained by slow diffusion of hexane $(20 \mathrm{~mL})$ into a concentrated solution of $\mathbf{4 b}$ in THF $(7 \mathrm{~mL})$ at -20 ${ }^{\circ} \mathrm{C}$. One of the crystals was used for an X-ray structure determination. Yield: $0.035 \mathrm{~g}$ (74\%). IR $\left(\mathrm{CH}_{2} \mathrm{Cl}_{2}, \mathrm{~cm}^{-1}\right): 1934(\mathrm{vs}), 1856(\mathrm{~s})\left(\mathrm{v}_{\mathrm{CO}}\right) .{ }^{1} \mathrm{H} \mathrm{NMR}\left(\mathrm{CD}_{2} \mathrm{Cl}_{2}\right): 9.01,8.41,7.92$ and 7.77 [m, 2H each,

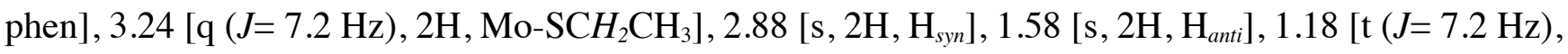
$\left.3 \mathrm{H}, \mathrm{Mo}-\mathrm{SCH}_{2} \mathrm{CH}_{3}\right], 0.62$ [s, 3H, $\mathrm{CH}_{3}$ of $\left.\eta^{3}-\mathrm{C}_{4} \mathrm{H}_{7}\right] .{ }^{13} \mathrm{C}\left\{{ }^{1} \mathrm{H}\right\} \mathrm{NMR}\left(\mathrm{CD}_{2} \mathrm{Cl}_{2}\right): 231.9[\mathrm{CO}], 152.0,138.1$, 136.3, 130.4, 127.6 and 125.3 [phen], $87.5\left[\mathrm{C}^{2}\right.$ of $\left.\eta^{3}-\mathrm{C}_{4} \mathrm{H}_{7}\right], 28.4$ [Mo- $\left.\mathrm{SCH}_{2} \mathrm{CH}_{3}\right], 21.1\left[\mathrm{Mo}-\mathrm{SCH}_{2} \mathrm{CH}_{3}\right]$, $19.3\left[\mathrm{CH}_{3}\right.$ of $\left.\eta^{3}-\mathrm{C}_{4} \mathrm{H}_{7}\right]$. The signals corresponding to $\mathrm{C}^{1}$ and $\mathrm{C}^{3}$ of the methallyl ligand are overlapped with the solvent residual peak, a fact clearly indicated by a 2D-HSQC experiment. Anal. Calcd. For $\mathrm{C}_{20} \mathrm{H}_{20} \mathrm{MoN}_{2} \mathrm{O}_{2} \mathrm{~S}: \mathrm{C}, 53.57 ; \mathrm{H}, 4.50 ; \mathrm{N}, 6.25$. Found: C, 53.66; H, 4.23; N, 5.96.

$\left[\mathbf{W}\left(\mathrm{SCH}_{2} \mathrm{CH}_{3}\right)\left(\eta^{3}-\mathbf{C}_{3} \mathbf{H}_{5}\right)(\mathbf{C O})_{2}(\right.$ phen $\left.)\right](5)$. The procedure was similar to that described above for the preparation of $\mathbf{3}$, starting from $\left[\mathrm{WCl}\left(\eta^{3}-\mathrm{C}_{3} \mathrm{H}_{5}\right)(\mathrm{CO})_{2}\right.$ (bipy)](0.100 g, $\left.0.201 \mathrm{mmol}\right), n \mathrm{BuLi}(0.15 \mathrm{~mL}$ of a 1.6 $\mathrm{M}$ solution in hexane, $0.242 \mathrm{mmol})$ and $\mathrm{EtSH}(18 \mu \mathrm{L}, 0.242 \mathrm{mmol})$. Compound 5 was obtained as a 
violet microcrystalline solid. Yield: $0.078 \mathrm{~g}(74 \%)$. IR $\left(\mathrm{CH}_{2} \mathrm{Cl}_{2}, \mathrm{~cm}^{-1}\right): 1925(\mathrm{vs}), 1837(\mathrm{~s})\left(\mathrm{v}_{\mathrm{CO}}\right) .{ }^{1} \mathrm{H}$ NMR $\left(\mathrm{CD}_{2} \mathrm{Cl}_{2}\right): 9.14,8.46,7.94$ and 7.76 [m, 2H each, phen], 3.34 [q $(\mathrm{J}=7.4 \mathrm{~Hz}), 2 \mathrm{H}, \mathrm{W}-\mathrm{SCH}_{2} \mathrm{CH}_{3}$, $2.99\left[\mathrm{~d}(J=6.2 \mathrm{~Hz}), 2 \mathrm{H}, \mathrm{H}_{s y n}\right], 2.22\left[\mathrm{~m}, 1 \mathrm{H}, \mathrm{H}_{\mathrm{c}}\right], 1.70\left[\mathrm{~d}(\mathrm{~J}=9.2 \mathrm{~Hz}), 2 \mathrm{H}, \mathrm{H}_{\text {anti }}\right], 1.17[\mathrm{t}(\mathrm{J}=7.4 \mathrm{~Hz}), 3 \mathrm{H}$, W-SCH$\left.{ }_{2} \mathrm{CH}_{3}\right] .{ }^{13} \mathrm{C}\left\{{ }^{1} \mathrm{H}\right\}$ NMR $\left(\mathrm{CD}_{2} \mathrm{Cl}_{2}\right): 224.7[\mathrm{CO}], 152.3,145.3,138.2,130.8,127.8$ and 125.7 [phen], 77.1 [C $\mathrm{C}^{2}$ of $\eta^{3}-\mathrm{C}_{3} \mathrm{H}_{5}$ ], $53.5\left[\mathrm{C}^{1}\right.$ and $\mathrm{C}^{3}$ of $\eta^{3}-\mathrm{C}_{3} \mathrm{H}_{5}$ ], $29.3\left[\mathrm{~W}-\mathrm{SCH}_{2} \mathrm{CH}_{3}\right], 21.3\left[\mathrm{~W}-\mathrm{SCH}_{2} \mathrm{CH}_{3}\right]$. Anal. Calcd. For $\mathrm{C}_{19} \mathrm{H}_{18} \mathrm{~N}_{2} \mathrm{O}_{2} \mathrm{SW}$ : C, 43.70; H, 3.47; N, 5.36. Found: C, 44.09; H, 3.23; N, 5.72.

$\left[\mathrm{Mo}\left(\mathbf{S C}_{6} \mathbf{H}_{5}\right)\left(\eta^{3}-\mathbf{C}_{4} \mathbf{H}_{7}\right)(\mathbf{C O})_{2}(\mathbf{b i p y})\right](\mathbf{6 a}) \cdot n \mathrm{BuLi}(0.15 \mathrm{~mL}$ of a $1.6 \mathrm{M}$ solution in hexane, $0.241 \mathrm{mmol})$ was added to a solution of $\mathrm{PhSH}(25 \mu \mathrm{L}, 0.241 \mathrm{mmol})$ in $\mathrm{THF}(10 \mathrm{~mL})$ at $-78{ }^{\circ} \mathrm{C}$. The resulting colorless solution was transferred using a cannula to a solution of $\left[\mathrm{MoCl}\left(\eta^{3}-\mathrm{C}_{4} \mathrm{H}_{7}\right)(\mathrm{CO})_{2}(\right.$ bipy $\left.)\right](0.080$ $\mathrm{g}, 0.201 \mathrm{mmol})$ in $\mathrm{THF}(15 \mathrm{~mL})$ previously cooled to $-78{ }^{\circ} \mathrm{C}$. The red color of the solution turned darker immediately. The solvent was evaporated to dryness under reduced pressure and the solid was extracted with $\mathrm{CH}_{2} \mathrm{Cl}_{2}(25 \mathrm{~mL})$. The resulting solution was filtered off the white solid (LiOTf) via cannula, concentrated under reduced pressure to a volume of $10 \mathrm{~mL}$, and addition of hexane caused the precipitation of compound $\mathbf{6 a}$ as a dark red solid, which was washed with hexane $(2 \times 15 \mathrm{~mL})$ and dried. Yield: $0.073 \mathrm{~g}(77 \%)$. IR (THF, $\left.\mathrm{cm}^{-1}\right): 1941(\mathrm{vs}), 1864(\mathrm{~s})\left(v_{\mathrm{CO}}\right) .{ }^{1} \mathrm{H}$ NMR $\left(\mathrm{CD}_{2} \mathrm{Cl}_{2}\right): 8.74,7.79$, 7.69 and $7.42[\mathrm{~m}, 2 \mathrm{H}$ each, bipy], $6.73[\mathrm{~m}, 1 \mathrm{H}, \mathrm{SPh}], 6.50[\mathrm{~m}, 2 \mathrm{H}, \mathrm{SPh}], 6.33[\mathrm{~m}, 2 \mathrm{H}, \mathrm{SPh}], 2.69[\mathrm{~s}$, $\left.2 \mathrm{H}, \mathrm{H}_{\text {syn }}\right], 1.48\left[\mathrm{~s}, 2 \mathrm{H}, \mathrm{H}_{\text {anti }}\right], 0.79\left[\mathrm{~s}, 3 \mathrm{H}, \mathrm{CH}_{3}\right.$ of $\left.\eta^{3}-\mathrm{C}_{4} \mathrm{H}_{7}\right] .{ }^{13} \mathrm{C}\left\{{ }^{1} \mathrm{H}\right\} \mathrm{NMR}\left(\mathrm{CD}_{2} \mathrm{Cl}_{2}\right): 230.1[C \mathrm{O}], 152.9$, 152.3, 137.6, 126.0 and 122.2 [bipy], 144.9, 134.0, 127.5 and 123.3 [SPh], 87.1 [C $\mathrm{C}^{2}$ of $\left.\eta^{3}-\mathrm{C}_{4} \mathrm{H}_{7}\right], 19.1$ $\left[\mathrm{CH}_{3}\right.$ of $\left.\eta^{3}-\mathrm{C}_{4} \mathrm{H}_{7}\right]$. The signals corresponding to $\mathrm{C}^{1}$ and $\mathrm{C}^{3}$ of the methallyl ligand are overlapped with the solvent residual peak, a fact clearly indicated by a 2D-HSQC experiment. Anal. Calcd. For $\mathrm{C}_{22} \mathrm{H}_{20} \mathrm{MoN}_{2} \mathrm{O}_{2} \mathrm{~S}: \mathrm{C}, 55.93 ; \mathrm{H}, 4.27 ; \mathrm{N}, 5.93$. Found: C, 56.25; H, 3.92; N, 6.00.

$\left[\mathrm{Mo}\left(\mathrm{SC}_{6} \mathbf{H}_{5}\right)\left(\eta^{3}-\mathbf{C}_{4} \mathbf{H}_{7}\right)(\mathbf{C O})_{2}(\mathbf{p h e n})\right](6 \mathrm{~b})$. The procedure was similar to that described above for the synthesis of $6 \mathbf{a}$, starting from $\left[\mathrm{MoCl}\left(\eta^{3}-\mathrm{C}_{4} \mathrm{H}_{7}\right)(\mathrm{CO})_{2}(\mathrm{phen})\right](0.075 \mathrm{~g}, 0.177 \mathrm{mmol}), n \mathrm{BuLi}(0.13 \mathrm{~mL}$ of a 1.6 M solution in hexane, $0.213 \mathrm{mmol})$ and $\mathrm{PhSH}(22 \mu \mathrm{L}, 0.213 \mathrm{mmol})$. Compound $\mathbf{6 b}$ was obtained as a red solid. Yield: $0.060 \mathrm{~g}(68 \%)$. IR (THF, $\left.\mathrm{cm}^{-1}\right): 1941(\mathrm{vs}), 1864(\mathrm{~s})\left(\mathrm{v}_{\mathrm{CO}}\right) .{ }^{1} \mathrm{H}$ NMR $\left(\mathrm{CD}_{2} \mathrm{Cl}_{2}\right): 9.06$, 8.28, 7.77 and 7.69 [m, 2H each, phen], 6.38 [m, 1H, SPh], $6.16[\mathrm{~m}, 2 \mathrm{H}, \mathrm{SPh}], 5.99$ [m, 2H, SPh], 2.82 $\left[\mathrm{s}, 2 \mathrm{H}, \mathrm{H}_{\text {syn }}\right], 1.56\left[\mathrm{~s}, 2 \mathrm{H}, \mathrm{H}_{\text {anti }}\right], 0.47\left[\mathrm{~s}, 3 \mathrm{H}, \mathrm{CH}_{3}\right.$ of $\left.\eta^{3}-\mathrm{C}_{4} \mathrm{H}_{7}\right] .{ }^{13} \mathrm{C}\left\{{ }^{1} \mathrm{H}\right\} \mathrm{NMR}\left(\mathrm{CD}_{2} \mathrm{Cl}_{2}\right): 229.7[C \mathrm{O}]$, $151.9,136.5,130.2,127.5,126.6$ and 125.0 [phen], 144.7, 143.9, 132.8 and 123.3 [SPh], 86.5 [C of $\left.\eta^{3}-\mathrm{C}_{4} \mathrm{H}_{7}\right], 19.1\left[\mathrm{CH}_{3}\right.$ of $\left.\eta^{3}-\mathrm{C}_{4} \mathrm{H}_{7}\right]$. The signals corresponding to $\mathrm{C}^{1}$ and $\mathrm{C}^{3}$ of the methallyl ligand are 
overlapped with the solvent residual peak, a fact clearly indicated by a 2D-HSQC experiment. Anal. Calcd. For $\mathrm{C}_{24} \mathrm{H}_{20} \mathrm{MoN}_{2} \mathrm{O}_{2} \mathrm{~S}$ : C, 58.06; H, 4.06; N, 5.64. Found: C, 58.26; H, 4.33; N, 5.13.

Synthesis of compound 7a. DMAD (17 $\mu \mathrm{L}, 0.141 \mathrm{mmol})$ was added to a solution of $\left[\mathrm{Mo}\left(\mathrm{SCH}_{2} \mathrm{CH}_{3}\right)\left(\eta^{3}-\mathrm{C}_{4} \mathrm{H}_{7}\right)(\mathrm{CO})_{2}\right.$ (bipy)](4a) $(50 \mathrm{mg}, 0.118 \mathrm{mmol})$, in $\mathrm{CH}_{2} \mathrm{Cl}_{2}$ at $-78{ }^{\circ} \mathrm{C}$. The solvent was evaporated under reduced pressure to a volume of $7 \mathrm{~mL}$, and the addition of hexane $(20 \mathrm{~mL})$ caused the precipitation of compound 7a as a dark red solid, which was washed with hexane $(2 \times 15 \mathrm{~mL})$, and dried in vacuo. Yield: $0.047 \mathrm{~g}(71 \%)$. IR $\left(\mathrm{CH}_{2} \mathrm{Cl}_{2}, \mathrm{~cm}^{-1}\right)$ : 1941(vs), 1860(s), 1702(w), 1698(w) (v $\left.\mathrm{v}_{\mathrm{CO}}\right)$. ${ }^{1} \mathrm{H}$ NMR $\left(\mathrm{CD}_{2} \mathrm{Cl}_{2}\right): 8.81,8.08,7.93$ and 7.44 [m, 2H each, bipy], 3.43 [s, 3H, OCH $], 2.87$ [s, 5H, $\mathrm{OCH}_{3}$ and $\mathrm{H}_{\text {syn }}$ ], 2.54 [q $\left.(J=7.2 \mathrm{~Hz}), 2 \mathrm{H}, \mathrm{SCH}_{2} \mathrm{CH}_{3}\right], 1.47\left[\mathrm{~s}, 2 \mathrm{H}, \mathrm{H}_{\text {anti }}\right], 1.17$ [t $(J=7.2 \mathrm{~Hz}), 3 \mathrm{H}$, $\left.\mathrm{SCH}_{2} \mathrm{CH}_{3}\right], 0.68$ [s, 3H, $\mathrm{CH}_{3}$ of $\left.\eta^{3}-\mathrm{C}_{4} \mathrm{H}_{7}\right] .{ }^{13} \mathrm{C}\left\{{ }^{1} \mathrm{H}\right\} \mathrm{NMR}\left(\mathrm{CD}_{2} \mathrm{Cl}_{2}\right): 229.4$ [CO], 199.5 [Mo-C(O)OCH $\mathrm{OH}_{3}$, $174.8\left[\mathrm{C}(\mathrm{O}) \mathrm{OCH}_{3}\right], 164.4[\mathrm{C}=\mathrm{C}], 153.5,153.0,137.9,125.3$ and 122.0 [bipy], $130.5[\mathrm{C}=\mathrm{C}], 86.0\left[\mathrm{C}^{2}\right.$ of $\left.\eta^{3}-\mathrm{C}_{4} \mathrm{H}_{7}\right], 57.0\left[\mathrm{C}^{1}\right.$ and $\mathrm{C}^{3}$ of $\left.\eta^{3}-\mathrm{C}_{4} \mathrm{H}_{7}\right], 52.1\left[\mathrm{OCH}_{3}\right], 50.9\left[\mathrm{OCH}_{3}\right], 28.9\left[\mathrm{SCH}_{2} \mathrm{CH}_{3}\right], 18.0\left[\mathrm{SCH}_{2} \mathrm{CH}_{3}\right]$, $13.7\left[\mathrm{CH}_{3}\right.$ of $\left.\eta^{3}-\mathrm{C}_{4} \mathrm{H}_{7}\right]$. Anal. Calcd. For $\mathrm{C}_{24} \mathrm{H}_{26} \mathrm{MoN}_{2} \mathrm{O}_{6} \mathrm{~S}: \mathrm{C}, 50.88 ; \mathrm{H}, 4.63 ; \mathrm{N}, 4.94$. Found: C, 51.10; H, 5.02; N, 4.69.

Synthesis of compound $\mathbf{7 b}$. Compound $\mathbf{7 b}$ was prepared as described above for $\mathbf{7 a}$, starting from $\left[\mathrm{Mo}\left(\mathrm{SCH}_{2} \mathrm{CH}_{3}\right)\left(\eta^{3}-\mathrm{C}_{4} \mathrm{H}_{7}\right)(\mathrm{CO})_{2}(\mathrm{phen})\right](4 \mathrm{~b})(36 \mathrm{mg}, 0.080 \mathrm{mmol})$ and DMAD $(12 \mu \mathrm{L}, 0.096 \mathrm{mmol})$. Compound $\mathbf{7 b}$ was obtained as dark red solid. The slow diffusion of hexane $(20 \mathrm{~mL})$ into a concentrated solution of $\mathbf{7 b}$ in $\mathrm{CH}_{2} \mathrm{Cl}_{2}(7 \mathrm{~mL})$ afforded dark red crystals, one of which was employed for the X-ray structure determination. Yield: $0.028 \mathrm{~g}$ (60\%). IR $\left(\mathrm{CH}_{2} \mathrm{Cl}_{2}, \mathrm{~cm}^{-1}\right)$ : 1941(vs), 1860(s), 1702(w), 1698(w) ( $\left.v_{\mathrm{CO}}\right) .{ }^{1} \mathrm{H}$ NMR $\left(\mathrm{CD}_{2} \mathrm{Cl}_{2}\right)$ : 9.20, 8.44, 7.93 and 7.79 [m, 2H each, phen], 3.35 [s, 3H, $\left.\mathrm{OCH}_{3}\right], 3.00\left[\mathrm{~s}, 2 \mathrm{H}, \mathrm{H}_{s y n}\right], 2.74\left[\mathrm{~s}, 3 \mathrm{H}, \mathrm{OCH}_{3}\right], 2.52\left[\mathrm{q}(\mathrm{J}=7.4 \mathrm{~Hz}), 2 \mathrm{H}, \mathrm{SCH}_{2} \mathrm{CH}_{3}\right], 1.54\left[\mathrm{~s}, 2 \mathrm{H}, \mathrm{H}_{\text {anti }}\right]$, $1.18\left[\mathrm{~m}, 3 \mathrm{H}, \mathrm{SCH}_{2} \mathrm{CH}_{3}\right], 0.36\left[\mathrm{~s}, 3 \mathrm{H}, \mathrm{CH}_{3}\right.$ of $\left.\eta^{3}-\mathrm{C}_{4} \mathrm{H}_{7}\right]$. The signal at $1.18 \mathrm{ppm}$ corresponding to the $\mathrm{CH}_{3}$ group of the SEt (listed as a multiplet) is overlapped with the signal of some hexane residual solvent. Anal. Calcd. For $\mathrm{C}_{26} \mathrm{H}_{26} \mathrm{MoN}_{2} \mathrm{O}_{6} \mathrm{~S}$ : C, 52.88; H, 4.44; N, 4.74. Found: C, 52.70; H, 4.29; N, 5.03 . 


\section{Results and discussion}

Neutral thiolato $\left[\mathrm{M}(\mathrm{SEt})(\mathrm{CO})_{3}(\mathrm{~N}-\mathrm{N})\right](\mathrm{M}=\mathrm{Mn}, \mathrm{Re} ; \mathrm{N}-\mathrm{N}=$ bipy, phen $)$ complexes are obtained by reaction of the corresponding triflato precursor with the equimolar amount of LiSEt (generated in situ from EtSH and BuLi in THF at $-78^{\circ} \mathrm{C}$ ) as depicted in Scheme 1. The reactions are instantaneous at -78 ${ }^{\circ} \mathrm{C}$, and accompanied by a change in the color of the solution from yellow to red. The IR spectra of the products display three intense $v_{\mathrm{CO}}$ bands, diagnostic of fac-tricarbonyl species, at frequencies significantly lower than those of the precursors (i.e. from 2044, 1953, $1938 \mathrm{~cm}^{-1}$ to 2003, 1912, 1902 $\mathrm{cm}^{-1}$ for compound 1). This is consistent with the substitution of the triflato ligands by thiolato groups, displaying a stronger donor ability that increases the electron density available for back-donation from the metal center to the carbonyl ligands. The new thiolato compounds $\mathbf{1}, \mathbf{2 a - b}$ were obtained as single products and could be isolated in good yields after a simple work up procedure (see Experimental Section). The new compounds are air sensitive, but they can be stored in the solid state under nitrogen several weeks.

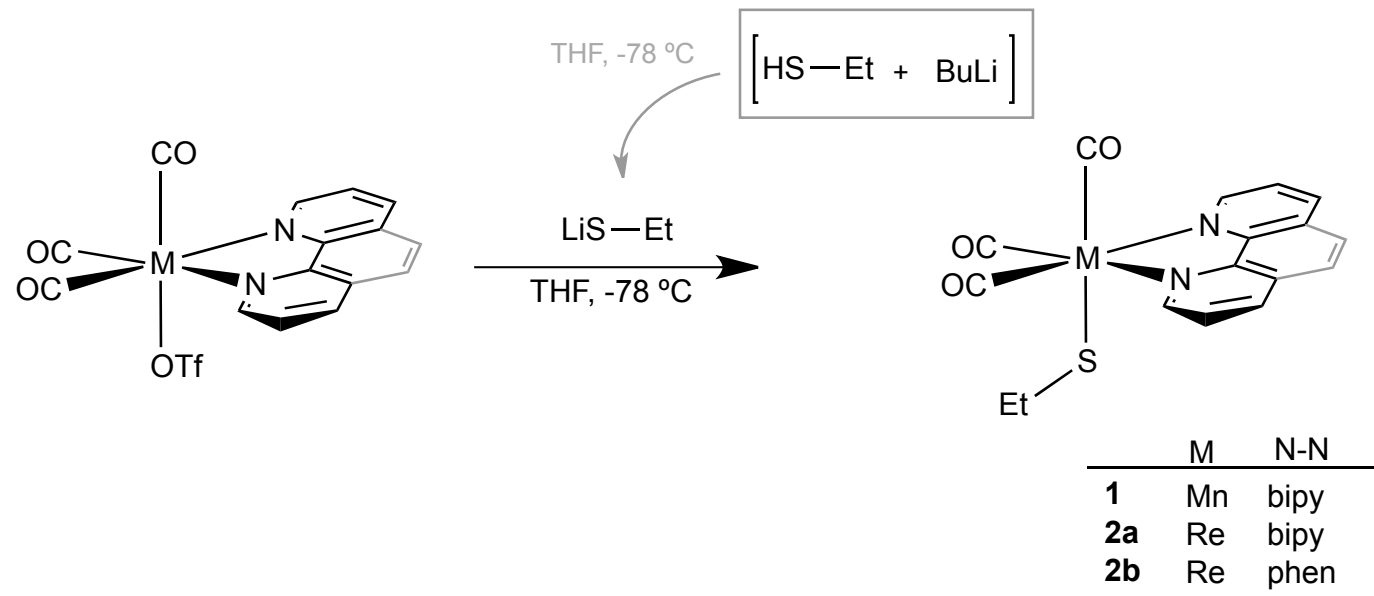

Scheme 1. Synthesis of ethylthiolato complexes $\left[M(S E t)(C O)_{3}(N-N)\right]$.

Compounds 1, 2a-b were fully characterized by means of NMR spectroscopy in solution, showing that the new species display the typical signals of $\mathrm{C}_{\mathrm{s}}$-symmetric geometry, $i$. $e$. four signals, of two hydrogens each, corresponding to a symmetric $\alpha$-diimine ligand in the ${ }^{1} \mathrm{H}$ NMR spectra, or two signals, one of double intensity of the other, for the three CO ligands in the ${ }^{13} \mathrm{C}$ NMR spectra. The incorporation of an ethylthiolato moiety for each $\left\{\mathrm{M}(\mathrm{CO})_{3}(\mathrm{~N}-\mathrm{N})\right\}$ fragment is clearly evidenced by the observation of a triplet and a quadruplet in the aliphatic zone (at 1.04 and $2.54 \mathrm{ppm}$ for compound $\mathbf{2 a}$, for example), that integrates for three and two hydrogen atoms, respectively, in the ${ }^{1} \mathrm{H}$ NMR spectrum.[38] 
The molecular structure of compound $\mathbf{2 a}$, determined by X-ray diffraction, is depicted in Figure 1 showing the rhenium atom in a distorted octahedral environment, in which the three carbonyl ligands are in $f a c$ disposition. Bond distances and angles around the metal centre are similar to those reported previously for complexes bearing the $\left\{\operatorname{Re}(\mathrm{CO})_{3}(\right.$ bipy $\left.)\right\}$ fragment,[39] in agreement with the solution IR and NMR data. In particular, the Re-S bond distance, of 2.486(5) $\AA$, is comparable to those of the only two mononuclear $\operatorname{Re}(\mathrm{I})$ thiolato complexes characterized by X-ray diffraction (Re-S bond lengths of 2.494(1) and 2.471(2) $\AA$, in the latter the thiolato group is forming part of a polydentate ligand).[39, 40] In fact, thiolato complexes of the ' $\mathrm{fac}$ - $\left[\operatorname{Re}(\mathrm{CO})_{3}\right]^{+}$' core have not been widely studied, in spite of their possible relevance in the context of HDS catalysis. [41, 42] Only a few examples are reported in the literature, [43-47] and the thiolato is usually acting as a bridging ligand. The average bond distance of Re-S, 2.525(4) $\AA$, in this type of binuclear complexes is, as can be expected, slightly longer than those mentioned above for terminal monomeric $\operatorname{Re}(\mathrm{I})$ thiolato complexes.[48]

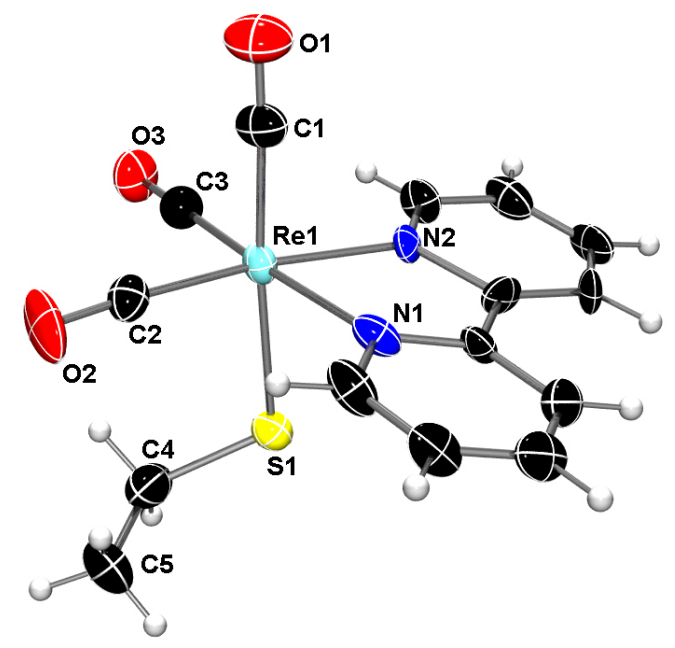

Figure 1. Molecular structure of complex 2 a, displaying thermal ellipsoids at $30 \%$ probability level.

The reaction of $\left[\mathrm{MoCl}\left(\eta^{3}\right.\right.$-allyl $\left.)(\mathrm{CO})_{2}(\mathrm{~N}-\mathrm{N})\right]\left(\eta^{3}\right.$-allyl $=\mathrm{C}_{3} \mathrm{H}_{5}, \mathrm{C}_{3} \mathrm{H}_{4}-2-\mathrm{Me} ; \mathrm{N}-\mathrm{N}=$ bipy, phen) with the equimolar amount of the in situ generated LiSEt led, in a few minutes at $-78{ }^{\circ} \mathrm{C}$, to the formation of the corresponding ethylthiolato complexes $\mathbf{3}, \mathbf{4 a - b}$ (see Scheme 2). The complexes were obtained as single products of the reactions, in good yields and were characterized spectroscopically in solution. 

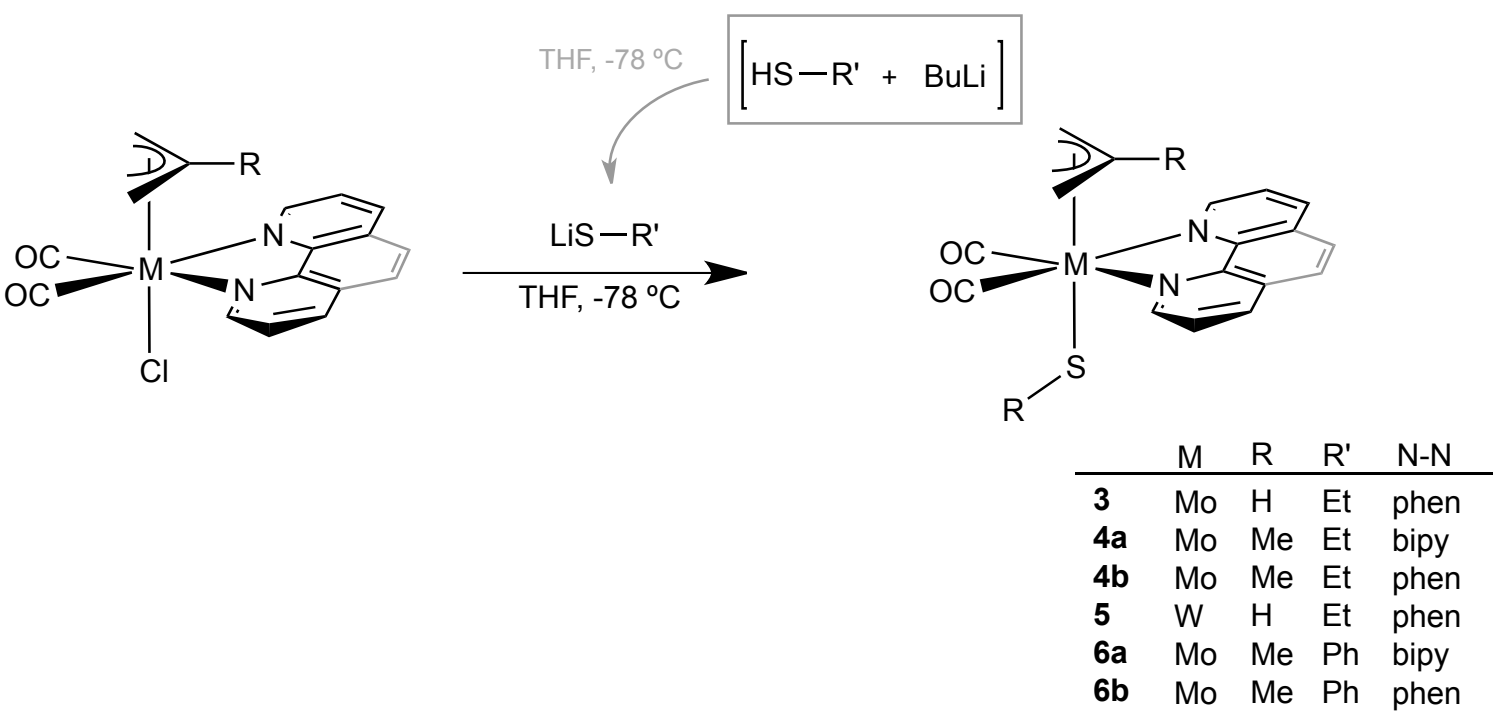

Scheme 2. Synthesis of thiolato complexes of $\left[M\left(\eta^{3}\right.\right.$-allyl $\left.)(\mathrm{CO})_{2}(\mathrm{~N}-\mathrm{N})\right]$ fragments.

The IR spectra in the carbonyl stretching region showed, in all cases, two intense bands, indicating the persistence of the cis- $\mathrm{Mo}(\mathrm{CO})_{2}$ unit, at lower wavenumbers than the starting chlorocomplexes. The ${ }^{1} \mathrm{H}$ NMR spectra clearly showed the presence of a molecular mirror plane as a set of four signals for bipy (or phen) hydrogens, and the equivalence of the two syn and of the two anti hydrogens of the $\eta^{3}$-allyl ligand are observed. The most distinctive feature in these spectra is the ethyl pattern, with a three-bonds $\mathrm{H}-\mathrm{H}$ coupling constant (of $7.4 \mathrm{~Hz}$ for complex $\mathbf{3}$, for example), indicative of the incorporation of the ethylthiolato ligand to the Mo(II) coordination sphere. The ${ }^{13} \mathrm{C}$ NMR spectra clearly showed the presence of the thiolato ligand (two new aliphatic carbon atom signals, at 28.4 and $20.9 \mathrm{ppm}$ for complex 3 , for example), and are in agreement with the proposed $\mathrm{C}_{\mathrm{s}}$ molecular symmetry, as only one signal is observed for the two carbonyl ligands, or for the two allyl terminal carbon atoms. Considering jointly all the spectroscopic features the geometry shown in Scheme 2 can be assigned to the new $\mathrm{Mo}$ (II) thiolato complexes. It must be noted that the thiolato complexes were found to be the only organometallic product of the reactions, and no product of the nucleophilic addition of the lithium thiolato to the $\eta^{3}$-allyl ligand was detected. The complex $\left[\mathrm{W}\left(\eta^{3}\right.\right.$-allyl $\left.)(\mathrm{SEt})(\mathrm{CO})_{2}(\mathrm{phen})\right](\mathbf{5})$ was synthesized analogously and isolated as a microcrystalline violet solid. IR and NMR data indicate that it is isostructural with the molybdenum counterparts (see Experimental Section for further experimental details). The new compounds are air sensitive, but they can be stored in the solid state under nitrogen several weeks. 
An X-ray structure determination showed that complex $\mathbf{4 b}$ consists of a molybdenum atom in a pseudooctahedral geometry (considering, as is usual for this type of complexes the allyl centroid occupying a coordination site), the thiolato and 2-methylallyl groups lying on opposite sides of the equatorial plane defined by the two carbonyls and the two nitrogens of the phen ligand. The allyl group is oriented with its open face pointing toward the carbonyl ligands, as is common to most known $\left[\mathrm{MoX}\left(\eta^{3}\right.\right.$-allyl $\left.)(\mathrm{CO})_{2}(\mathrm{~N}-\mathrm{N})\right]$ complexes.[49, 50] The Mo1-S1 bond distance, of 2.460(2) $\AA$ is slightly shorter than that found in the only $\left\{\mathrm{Mo}\left(\eta^{3}\right.\right.$-allyl $\left.)(\mathrm{CO})_{2}\right\}$ thiolato complex characterized by $\mathrm{X}$-ray diffraction, a hydrosulfide complex with a Mo-S bond length of 2.506(1) A..[51] Only a small number of monomeric Mo(II) thiolato compounds are known, a situation that may be due to the strong tendency of thiolato ligands to coordinate as bridges, and therefore to form polymetallic structures. In fact, mononuclear, low-valent molybdenum compounds of monodentate thiolato are very scarce in the literature.[22, 52-60] We therefore extended our study, and Mo(II) benzenethiolato complexes 6a-b were obtained following the same synthetic strategy depicted in Scheme 2, using PhSH instead of EtSH. The new arylthiolato compounds were obtained in good yields, and their spectroscopic data are in agreement with the geometry shown in Scheme 2.

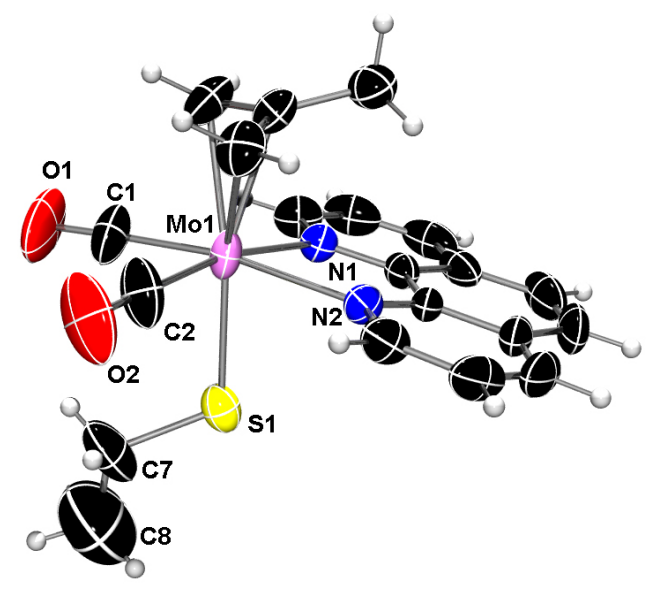

Figure 2. Molecular structure of compound $\mathbf{4 b}$, displaying thermal ellipsoids at $30 \%$ probability level.

We had previously found that the reactivity of $\left[\mathrm{MoX}\left(\eta^{3}-\right.\right.$ allyl $\left.)(\mathrm{CO})_{2}(\mathrm{~N}-\mathrm{N})\right]$ and $\left[\mathrm{ReX}(\mathrm{CO})_{3}(\mathrm{~N}-\mathrm{N})\right](\mathrm{X}=$ alkoxo,[25, 26] hydroxo [51, 61, 62] or amido,[27, 63-65]) complexes towards organic electrophiles is dominated by the nucleophilicity of the $\mathrm{X}$ ligand. This is attributed to the electronic conflict between the lone pairs on $\mathrm{X}$ and the filled $\mathrm{d} \pi$ metal orbitals, and to the high polarity of the metal-X bond. With this in mind we decided to explore the reactivity of the new thiolato molybdenum compounds towards unsaturated organic electrophiles. The reaction of ethylthiolato complexes 4a,b with 
dimethylacetylenedicarboxylate (DMAD) in $\mathrm{CH}_{2} \mathrm{Cl}_{2}$ at low temperature afforded immediately the corresponding insertion compounds $\mathbf{7 a , b}$ respectively, as the only products of the reactions (see Scheme 3).
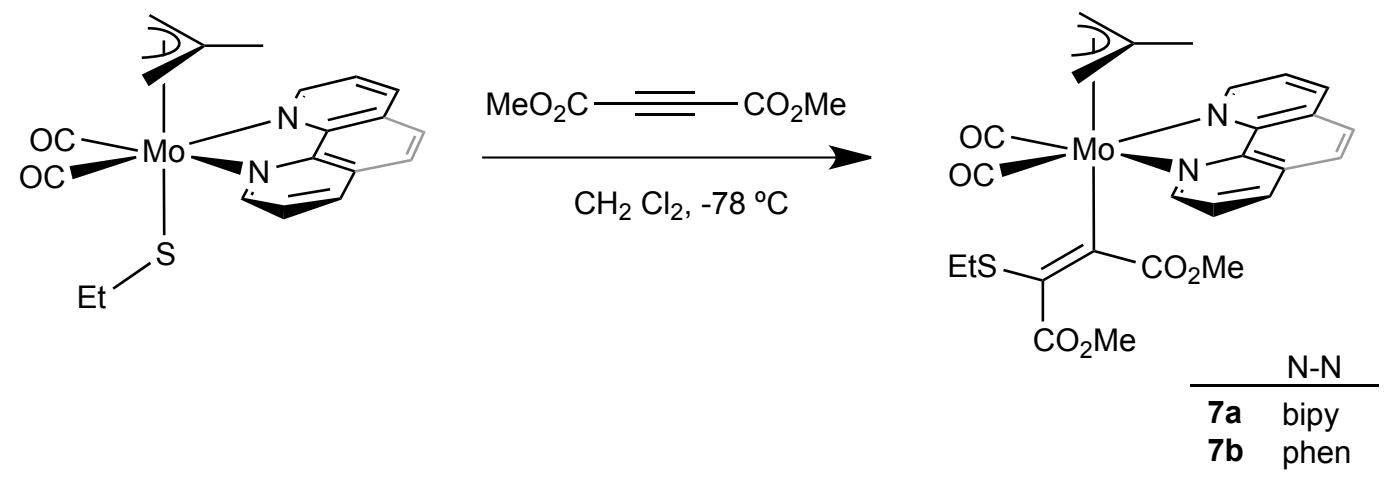

Scheme 3. Reactivity of $\left[M o\left(\eta^{3}\right.\right.$-methallyl $\left.)(S E t)(C O)_{2}(N-N)\right](6 a, b)$ compounds towards DMAD.

Slow diffusion of hexane into a concentrated solution of $7 \mathbf{b}$ in $\mathrm{CH}_{2} \mathrm{Cl}_{2}$ at $-20{ }^{\circ} \mathrm{C}$ afforded red crystals, one of which was used for an X-ray structure determination. The results showed, as depicted in Figure 3 , that in complex $\mathbf{7 b}$ the coordination geometry around the molybdenum can be described as pseudooctahedral, with the allyl centroid and the two $\mathrm{CO}$ defining one face of the octahedron. The $\mathrm{N}$ atoms of the phen are trans to the CO ligands, whereas trans to the allyl is the Z-alkenyl ligand resulting from the formal insertion of DMAD into the Mo-S bond. The Mo1-C9 and C9-C10 bond lengths, of 2.257(10) and 1.359(14) respectively, along with the bond angles around C9 and C10 (confirming their $\mathrm{sp}^{2}$ hybridization) are indicative of the alkenyl character of the new ligand.

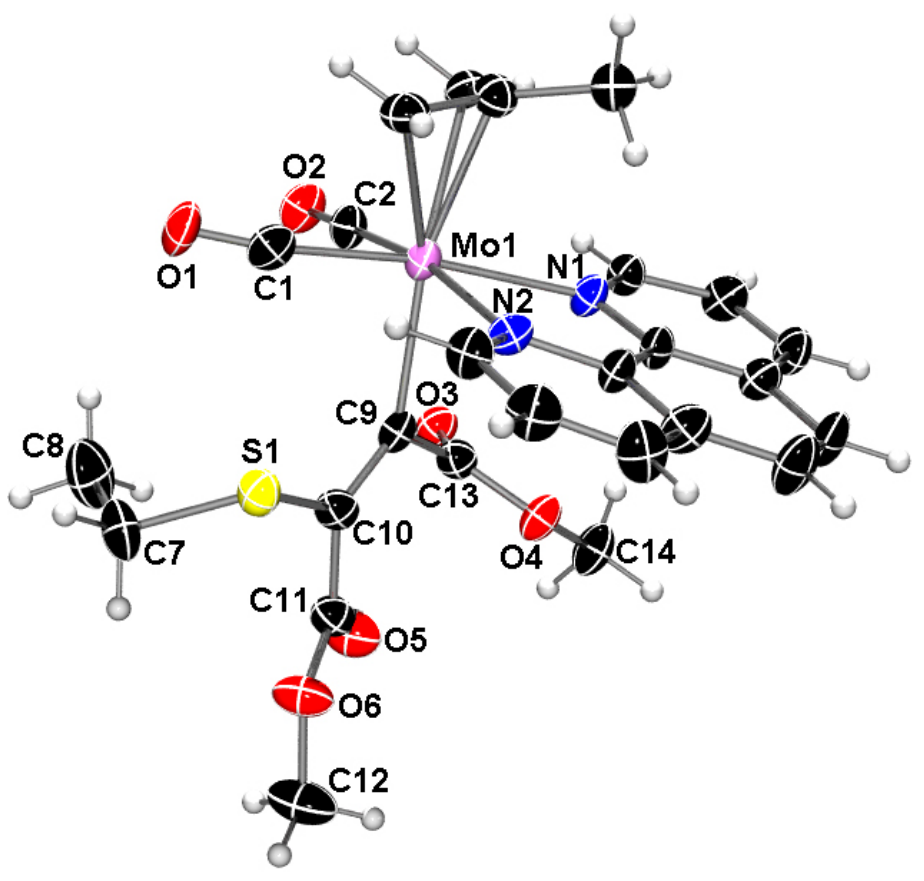

Figure 3. Molecular structure of the insertion product $\mathbf{7 b}$, displaying thermal ellipsoids at $30 \%$ probability level. 
The spectroscopic data in solution of the Z-alkenyl compounds $\mathbf{7 a , b}$ are in accordance with the structure found in the solid state. In the carbonyl region of the IR spectra, apart from the two intense bands corresponding to the two Mo-CO ligands (at 1940 and $1860 \mathrm{~cm}^{-1}$ for $7 \mathbf{a}$, for example), two weaker bands (at 1702, 1698 for both 7a and 7b) are observed, corresponding to the ester CO groups (free DMAD displays a $v_{\mathrm{CO}}$ band at $1727 \mathrm{~cm}^{-1}$ ). In the ${ }^{1} \mathrm{H}$ NMR spectra of 7a,b the incorporation of a DMAD molecule is clearly observed, as two non-equivalent methoxy groups are present (at 3.43 and 2.87 ppm for 7a), while the signal pattern of the ethylthiolato precursors $\mathbf{4 a}, \mathbf{b}$ is maintained (i.e. $\mathrm{C}_{\mathrm{s}}$ symmetry is observed). The ${ }^{13} \mathrm{C}$ NMR spectra indicate the formation of a Z-alkenyl ligand, as two ester CO carbon atoms are observed (two low intensity signals at 199.5 and $174.8 \mathrm{ppm}$ for $7 \mathbf{a}$ ), as well as the signals corresponding to the two olefinic carbon atoms (at 164.4 and $130.5 \mathrm{ppm}$ for $7 \mathbf{a}$ ). This reactivity is reminiscent of that found for the analogous molybdenum alkoxo complexes, [26] and as happened for those, the lack of a vacant coordination site in the metal complex (or a labile ligand), allows us to propose a direct nucleophilic attack to the activated alkyne by the undissociated thiolato ligand as the most plausible initial step in the mechanism for the insertion reaction.

The phenylthiolato complexes $\mathbf{6 a , b}$ failed to react with DMAD, which can be attributed to the less nucleophilic character of sulfur atom in these ligands, in turn due to the less electron donating character of aryls in comparison with alkyl groups. Indeed, this fact is clearly observed when the IR spectra (CO stretching region) of the phenylthiolato complexes are compared to those of the ethylthiolato analogs. For example, $v_{\mathrm{CO}}$ bands for ethylthiolato $4 \mathbf{a}$ are observed at 1933 and $1855 \mathrm{~cm}^{-1}$, whereas for the phenylthiolato 6a they occur at significantly higher wavenumbers, 1941 and $1864 \mathrm{~cm}^{-1}$.

\section{Conclusions}

New terminal mononuclear thiolato complexes of the widely used $f a c-\left\{\mathrm{M}(\mathrm{CO})_{3}(\mathrm{~N}-\mathrm{N})\right\}(\mathrm{M}=\mathrm{Mn}, \mathrm{Re}$; $\mathrm{N}-\mathrm{N}=$ bipy, phen) fragments have been obtained by simple reaction of the triflato precursors with lithium ethanethiolato. The new complexes have been fully characterized in solution (IR, NMR spectroscopies), and in the case of the $\left[\operatorname{Re}(\mathrm{SEt})(\mathrm{CO})_{3}(\right.$ bipy $\left.)\right](\mathbf{4 a})$ the solid-state structure has been

determined by X-ray diffraction. Under similar mild conditions (THF and low temperature), alkaneand arylthiolato $\left[\mathrm{Mo}(\mathrm{SR})\left(\eta^{3}\right.\right.$-allyl $\left.)(\mathrm{CO})_{2}(\mathrm{~N}-\mathrm{N})\right](\mathrm{M}=\mathrm{Mo}, \mathrm{W} ; \mathrm{R}=\mathrm{Et}, \mathrm{Ph} ; \mathrm{N}-\mathrm{N}=$ bipy, phen) compounds have been obtained, by a methatesis of the chloride by the triflato ligand from the corresponding chlorocomplexes. The ethylthiolato complexes $(\mathbf{4 a}, \mathbf{b})$ showed a notably higher reactivity towards the unsaturated alkyne DMAD, affording Z-alkenyl products $(\mathbf{7 a}, \mathbf{b})$ as a result of the insertion of the 
alkyne into the Mo-S bond. The X-ray structure of one insertion product (7b) as well as that of its thiolato precursor $(\mathbf{4 b})$ have been determined.

\section{Acknowledgments}

Financial support from Ministerio de Economía y Competitividad/FEDER (grants PGC2018-097366B-100 and CTQ2015-70231-P) and Principado de Asturias (grant GRUPIN14-103, and Severo Ochoa

predoctoral fellowship to S. F.) is gratefully acknowledged. S. Z. participated in this work during her stay at Oviedo University as an Erasmus exchange student from Heidelberg University.

\section{References}

[1] I. G. Dance, Polyhedron 5 (1986), 1037-1104. https://doi.org/10.1016/S0277-5387(00)84307-7

[2] P. J. Blower, J. R. Dilworth, Coord. Chem. Rev. 76 (1987), 121-185. https://doi.org/10.1016/0019$\underline{8545(87) 85003-8}$

[3] M. C. Gimeno, Handbook of Chalcogen Chemistry; Royal Society of Chemistry: London, 2007; Chapter 2.1, pp 33-80. https://doi.org/10.1039/9781847557575-00031

[4] G. Henkel, B. Krebs, Chem. Rev. 104 (2004), 801-824. https://doi.org/10.1021/cr020620d

[5] R. H. Holm, P. Kennepohl, E. I. Solomon, Chem. Rev. 96 (1996), 2239-2314. https://doi.org/10.1021/cr9500390

[6] D. Coucouvanis, Acc. Chem. Res. 24 (1991), 1-8. https://doi.org/10.1021/ar00001a001

[7] A. A. Mohamed, H. E. Abdou, J. Chen, A. E. Brice, M. R. M. Bruce, Comments Inorg. Chem. 23 (2002), 321-334. https://doi.org/10.1080/02603590215003

[8] C. F. Shaw, Chem. Rev. 99 (1999), 2589-2600. https://doi.org/10.1021/cr9804310

[9] M. A. Malik, Comprehensive Inorganic Chemistry II 4 (2013), 177-210.

https://doi.org/10.1016/B978-0-08-097774-4.00411-3

[10] M. Afzaal, M. A. Malik, P. O’Brien, J. Mat. Chem. 20 (2010), 4031-4040.

https://doi.org/10.1039/B923898K

[11] T. V. Choudhary, Ind. Eng. Chem. Res. 46 (2007), 8363-8370. https://doi.org/10.1021/ie061617d

[12] S. Brunet, D. Mey, G. Pérot, C. Bouchy, F. Diehl, Appl. Catal., A 278 (2005), 143-172.

https://doi.org/10.1016/j.apcata.2004.10.012 
[13] J. Ancheyta, M. S. Rana, E. Furimsky, Catal. Today 109 (2005), 3-15.

https://doi.org/10.1016/j.cattod.2005.08.025

[14] D. Stirling, The Sulfur Problem: Cleaning up Industrial Feedstocks, RSC Clean Technology Monographs, Royal Society of Chemistry, Cambridge, UK, 2000.

[15] A. N. Startsev, I. I. Zalharov, Russ. Chem. Rev. 72 (2003), 517-536.

http://dx.doi.org/10.1070/RC2003v072n06ABEH000772

[16] R. A. Sánchez-Delgado, Organometallic Modeling of the Hydrodesulfurization and

Hydrodenitrogenation Reactions, Kluwer Academic Publishers, Boston, 2002.

[17] R. A. Sánchez-Delgado, Comprehensive Organometallic Chemistry III, R. H. Crabtree, D. M. P. Mingos (Eds.), Elsevier, Oxford, 2006, Vol.1, Chapter 27.

[18] R. J. Angelici, Organometallics 20 (2001), 1259-1275. https://doi.org/10.1021/om010040r

[19] R. J. Angelici, Polyhedron 16 (1997) 3073-3088. https://doi.org/10.1016/S0277-5387(96)00581-5

[20] C. Bianchini, A. Meli, F. Vizza, J. Organomet. Chem. 689 (2004), 4277-4290.

https://doi.org/10.1016/j.jorganchem.2004.05.027

[21] A. Sattler, G. Parkin, J. Am. Chem. Soc. 133 (2011), 3748-3751.

https://doi.org/10.1021/ja111034g

[22] D. Buccella, K. E, Janak, G. Parkin, J. Am. Chem. Soc. 130 (2008), 16187-16189.

https://doi.org/10.1021/ja8070545

[23] J. Pérez, L. Riera, V. Riera, S. García-Granda, E. García-Rodríguez, J. Am. Chem. Soc. 123

(2001), 7469-7470. https://doi.org/10.1021/ja015538z

[24] J. Pérez, L. Riera, V. Riera, S. García-Granda, E. García-Rodríguez, D. Miguel, Organometallics 21 (2002), 1622-1626. https://doi.org/10.1021/om0107800

[25] E. Hevia, J. Pérez, L. Riera, V. Riera, I. Del Río, S. García-Granda, D. Miguel, Chem. Eur. J. 8 (2002), 4510-4521. https://doi.org/10.1002/1521-3765(20021004)8:19<4510::AID-

CHEM4510>3.0.CO;2-L

[26] E. Hevia, J. Pérez, L. Riera, V. Riera, D. Miguel, Organometallics 21 (2002), 1750-1752. https://doi.org/10.1021/om010977s

[27] D. Morales, J. Pérez, L. Riera, V. Riera, D. Miguel, M. E. G. Mosquera, S. García-Granda, Chem. Eur. J. 9 (2003), 4132-4143. https://doi.org/10.1002/chem.200304981

[28] E. Hevia, J. Pérez, V. Riera, D. Miguel, Organometallics 21 (2002), 1966-1974.

https://doi.org/10.1021/om0110383 
[29] B. J. Brisdon, G. F. Griffin, J. Chem. Soc., Dalton Trans. (1975), 1999-2002.

https://doi.org/10.1039/DT9750001999

[30] Triflato complexes of this kind have been previously prepared, see for instance: J. Guerrero, O. E. Piro, E. Wolcan, M. R. Feliz, G. Ferraudi, S. A. Moya, Organometallics 20 (2001), 2842-2853. https://doi.org/10.1021/om000784p

[31] R. M. Carlos, I. Ap. Carlos, B. S. L. Neto, M. G. Neumann, Inorg. Chim. Acta 299 (2000), 231237. https://doi.org/10.1016/S0020-1693(99)00505-8

[32] SAINT+. SAX area detector integration program. Version 6.02. Bruker AXS, Inc. Madison, WI, 1999.

[33] G. M. Sheldrick, SADABS, Empirical Absorption Correction Program. University of Göttingen: Göttingen, Germany, 1997.

[34] CrysAlis ${ }^{\text {Pro }}$ CCD, CrysAlis ${ }^{\text {Pro }}$ RED. Oxford Diffraction Ltd., Abingdon, Oxfordshire, UK.

[35] G. M. Sheldrick, Acta Crystallogr. A64 (2008), 112-122.

https://doi.org/10.101107/S0108767307043930

[36] O. V. Dolomanov, L. J. Bourhis, R. J. Gildea, J. A. K. Howard, H. Puschmann, J. Appl. Cryst. 42 (2009), 339-341. https://doi.org/10.101107/S0021889808042726

[37] L. J. Farrugia, J. Appl. Cryst. 30 (1997), 565. https://doi.org/10.101107/S0021889897003117

[38] Complex 2a had been previously synthesized in our group as the product of the reaction of the amido $\left[\mathrm{Re}(\mathrm{NHp}-\mathrm{Tol})(\mathrm{CO})_{3}(\right.$ bipy $\left.)\right]\left(\mathrm{p}-\mathrm{Tol}=4-\mathrm{MeC}_{6} \mathrm{H}_{4}\right)$ with $\mathrm{EtSH}$, see ref 28.

[39] V. Fernández-Moreira, H. Sastre-Martín, Inorg. Chim. Acta 460 (2017), 127-133.

https://doi.org/10.1016/j.ica.2016.07.038

[40] D. J. Kramer, A. Davison, W. M. Davis, A. G. Jones, Inorg. Chem. 41 (2002), 6181-6183. https://doi.org/10.1021/ic025887+

[41] R. D. Adams, P. Dhull, J. D. Tedder, Inorg. Chem. 57 (2018), 7957-7965.

https://doi.org/10.1021/acs.inorgchem.8b01091

[42] T. A. Pecorato, R. R. Chianelli, J. Catal. 67 (1981), 430-445. https://doi.org/10.1016/00219517(81)90303-1

[43] M. He, H. Y. V. Ching, C. Policar, H. C. Bertrand, New J. Chem. 42 (2018), 11312-11323. https://doi.org/10.1039/C8NJ01960F

[44] Y. Chen, L. Zhang, Z. Chen, Acta Crystallogr. E58 (2002), m590-m591.

https://doi.org/10.1107/S1600536802017129 
[45] V. W.-W. Yam, Y.-L. Pui, K. M.-C. Wong, K.-K. Cheung, Chem. Commun. (2000), 1751-1752. https://doi.org/10.1039/B005092J

[46] V. W.-W. Yam, K. M.-C. Wong, K.-K. Cheung, Organometallics 16 (1997), 1729-1734. https://doi.org/10.1021/om960797w

[47] W.-H. Leung, T. S. M. Hun, S. Fung, I. D. Williams, K.-Y. Wong, Polyhedron 16 (1997), 36413648. https://doi.org/10.1016/S0277-5387(97)00098-3

[48] This fact has been already observed, see: Md. M. Hossain, H.-M. Lin, S.-G. Shyu, Organometallics 22 (2003), 3262-3270, and references therein. https://doi.org/10.1021/om030131j

[49] M. J. Calhorda, P. J. Costa, Coord. Chem. Rev. 344 (2017), 83-100. https://doi.org/10.1016/j.ccr.2016.12.010

[50] D. E. Ryan, D. J. Cardin, F. Hartl, Coord. Chem. Rev. 335 (2017), 103-149. https://doi.org/10.1016/j.ccr.2016.12.018

[51] D. C. Gerbino, E. Hevia, D. Morales, M. E. N. Clemente, J. Pérez, L. Riera, V. Riera, D. Miguel, Chem. Commun. (2003), 328-329. https://doi.org/10.1039/B210860G

[52] Roknuzzaman, Sm. M. T. Abedin, M. R. Haque, S. Ghosh, D. A. Tocher, M. G. Richmond, S. E. Kabir, Polyhedron 164 (2019), 55-63. https://doi.org/10.1016/j.poly.2019.02.23

[53] M. R. Haque, S. Ghosh, G. Hograth, M. G. Richmond, S. E. Kabir, Inorg. Chimica Acta 434 (2015), 150-157. https://doi.org/10.1016/j.ica.2015.05.010

[54] K.-H. Yih, G.-H. Lee, Y. Wang, Inorg. Chem. 42 (2003), 1092-1100.

https://doi.org/10.1021/ic020579x.

[55] D. C. Povey, R. L. Richards, C. Shortman, Polyhedron 5 (1986), 369-376.

https://doi.org/10.1016/S0277-5387(00)84935-9

[56] P. M. Treichel, P. C. Nakagaki, Organometallics 5 (1986), 711-716.

https://doi.org/10.1021/om00135a014

[57] M. Kamata, T. Yoshida, S. Otsuka, K. Hirotsu, T. Higuchi, J. Am. Chem. Soc. 103 (1981), 35723574. https://doi.org/10.1021/ja00402a054

[58] S. Otsuka, M. Kamata, K. Hirotsu, T. Higuchi, J. Am. Chem. Soc. 103 (1981), 3011-3014. https://doi.org/10.1021/ja00401a017

[59] J. Chatt, J. P. Lloyd, R. L. Richards, J. Chem. Soc., Dalton Trans. (1976), 565-568. https://doi.org/10.1039/DT9760000565

[60] D. D. Watkins Jr., T. A. George, J. Organomet. Chem. 102 (1975), 71-77.

https://doi.org/10.1016/S0022-328X(00)90262-6 
[61] L. Cuesta, E. Hevia. D. Morales, J. Pérez, L. Riera, D. Miguel, Organometallics 25 (2006), 17171722. https://doi.org/10.1021/om051081g

[62] L. Cuesta, D. C. Gerbino, E. Hevia, D. Morales, M. E. N. Clemente, J. Pérez, L. Riera, V. Riera, D. Miguel, I. Del Río, S. García-Granda, Chem. Eur. J. 10 (2004), 1765-1777.

https://doi.org/10.1002/chem.200305577

[63] E. Hevia, J. Pérez, V. Riera, D. Miguel, Organometallics 22 (2003), 257-263.

https://doi.org/10.1021/om020667p

[64] D. Morales, J. Pérez, L. Riera, V. Riera, D. Miguel, Inorg. Chem. 41 (2002), 4111-4113.

https://doi.org/10.1021/ic025665b

[65] E. Hevia, J. Pérez, V. Riera, D. Miguel, Chem. Commun. (2002), 1814-1815.

https://doi.org/10.1039/B203261A

[66] A $\eta^{3}$ - to $\eta^{1}$ - rearrangement of the methallyl ligand and coordination of the DMAD molecule to the Mo atom can not be discarded.

\section{GRAPHICAL ABSTRACT}

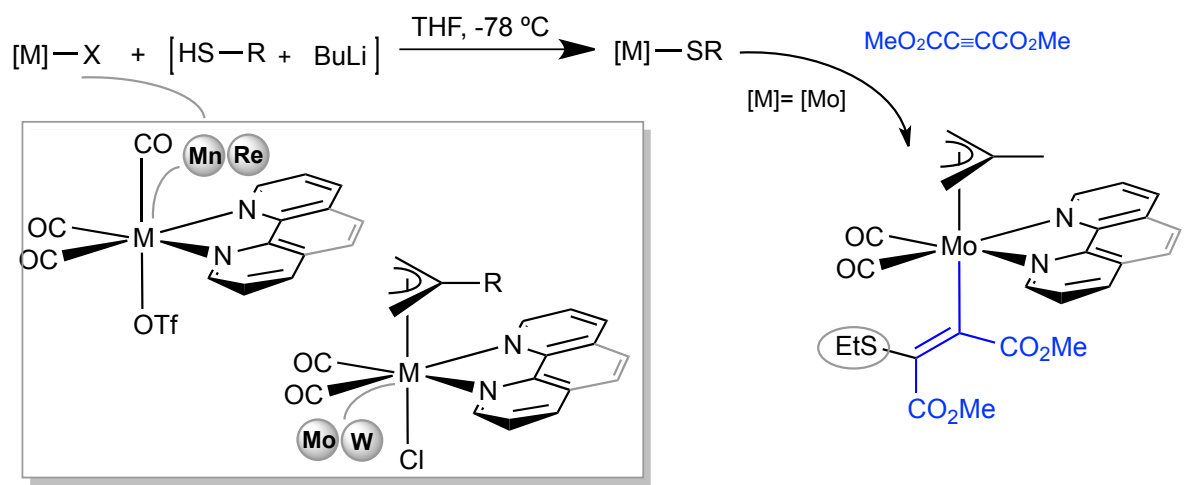

New terminal mononuclear thiolato complexes of the widely used $f a c-\left\{\mathrm{M}(\mathrm{CO})_{3}(\mathrm{~N}-\mathrm{N})\right\}(\mathrm{M}=\mathrm{Mn}, \mathrm{Re})$ and cis- $\left\{\mathrm{Mo}\left(\eta^{3}\right.\right.$-allyl $\left.)(\mathrm{CO})_{2}(\mathrm{~N}-\mathrm{N})\right](\mathrm{M}=\mathrm{Mo}, \mathrm{W})$ fragments, in which $\mathrm{N}-\mathrm{N}=$ bipy or phen, have been easily synthesized. The more reactive ethylthiolato molybdenum complexes smoothly insert a dimethylacetylenedicarboxylate molecule into the Mo-S bond, affording $Z$-alkenyl products. 